\title{
The effect of response-contingent feedback stimuli under two types of avoidance extinction conditions
}

\author{
CYNTHIA SCHEUER \\ Florida Atlantic University, Boca Raton, Florida 33432 \\ and \\ LAWRENCE I. SCHONFELD \\ University of South Florida, Tampa, Florida 33620
}

\begin{abstract}
Four groups of rats were conditioned to avoid shock in a two-way shuttlebox. During acquisition, each response both precluded a brief programmed shock and produced a 1-sec feedback signal. CS termination was not permitted during either acquisition or extinction. During extinction, response-produced or yoked feedback was combined with either $100 \%$ response-independent shock or no-shock conditions. Greatest resistance to extinction of avoidance behavior was obtained when response-produced feedback occurred in the absence of shock relative to any other combination of these events. These results, when compared to those of previous studies investigating the role of warning signal termination in combination with the presence or absence of shock in extinction (Hartley, 1968), clearly reveal a functional similarity between feedback and CS termination.
\end{abstract}

The most prevalent interpretation of discriminative avoidance behavior has been the two-factor theory, originally discussed by Mowrer and Lamoreaux (1946) and more recently by Solomon and Brush (1956). According to the theory, conditioning is accomplished by both Pavlovian and operant processes. A classically conditioned fear response develops by repeated pairings of CS and US. This fear reaction motivates the subject to avoid the US. Reinforcement of avoidance occurs when fear is reduced by response-produced termination of the CS and preclusion of the US.

An alternative explanation of avoidance views the CS, CS termination, and the presence or absence of the US as having discriminative rather than motivational properties (Mowrer \& Jones, 1945; Sheffield, 1949). When one or all of these cues are altered during extinction, avoidance behavior declines due to generalization decrement. Thus, if a subject is avoiding either a high proportion of shocks during terminal acquisition or all of them, a no-shock extinction procedure should prolong extinction over a procedure in which shocks occur on all extinction trials. Similarly, any extinction procedure that alters the consequences of responding from those occurring during the acquisition phase, whether the events be CS termination, US avoidance, or any other highly discriminable events, should facilitate extinction of the behavior.

This study was a portion of a thesis submitted by the second author in partial fulfillment of the requirements for the degree of Master of Arts in psychology at Florida Atlantic University.
In line with a generalization decrement interpretation of avoidance, D'Amato, Fazzaro, and Etkin (1968) have proposed that response-produced CS termination serves not necessarily to inhibit fear, but rather, as a discriminative feedback signal for successful avoidance. They have demonstrated that a response-produced feedback stimulus, never paired with shock onset or offset, can facilitate acquisition of avoidance behavior in a manner similar to that of response-produced CS termination. Whether the function of feedback is to provide the subject with information regarding the nonoccurrence of shock or it acts as a conditioned inhibitor of fear or serves both functions is as yet unresolved.

Bolles, Moot, and Grossen (1971) compared the removal of either feedback or CS termination during extinction of avoidance behavior. Their results demonstrated that either contingency can serve to maintain avoidance behavior in extinction, but only in the absence of further shock. Katzev and Henderson (1971) have suggested that response-contingent feedback will serve to maintain avoidance behavior only if it is not a redundant predictor of US avoidance. In the Bolles et al. study, acquisition was accomplished under conditions in which feedback was redundant and occurred simultaneously with CS termination. Thus, either event could have been a redundant cue for some of the animals. While there have been numerous demonstrations of the functional similarity between response-produced feedback and warning signal termination during acquisition of avoidance (Bolles \& Grassen, 1969; Cicala \& Owen, 
1976; D'Amato et al., 1968; Galvani \& Twitty, 1978), there remains little evidence of its effects during extinction of discriminated avoidance behavior. Although Cicala and Owen found that response-produced warning signal termination resulted in greater resistance to extinction of avoidance than response-produced feedback (Cicala \& Owen, 1976, Experiment 3), their results do not, as Galvani and Twitty (1978) recently remarked, necessarily imply different functional properties of the two. First of all, the relative "salience" of each type of event may have contributed to differential extinction rates, that is, their feedback stimulus, light offset, may have been less salient than noise offset for their rats. In effect, they failed to counterbalance the type of stimulus change that served as the CS or feedback cue. Furthermore, no attempt was made to differentiate between response-produced and response-independent feedback on resistance to extinction.

The present experiment was designed to investigate the role of a response-produced vs. response-independent feedback signal in the absence of warning signal termination during extinction of avoidance. In an earlier study, Hartley (1968) found that an extinction procedure that incorporated response-contingent termination of the warning signal in combination with US nonoccurrence produced greater resistance to extinction than any other combination of these events. We attempted to determine whether response-produced feedback signal in combination with shock presence or absence interacted in a manner similar to that found for CS termination.

\section{METHOD}

\section{Subjects}

The subjects were 24 male albino rats obtained from the Holtzman Company, ranging in age from 76 to 89 days. They were housed individually, with food and water continuously available in the home cage.

\section{Apparatus}

The apparatus consisted of two modified Mowrer-Miller shuttleboxes with inside dimensions of $61 \times 15.2 \times 23 \mathrm{~mm}$ high. The top lid and ends of each box were painted flat black, while the sides were clear 6-mm Plexiglas. The floor consisted of $453-\mathrm{mm}$ brass bars, $1.3 \mathrm{~cm}$ apart from center to center. A $2.5 \times 14-\mathrm{cm}$ brass footplate was located in the center of the boxes. A hinge was located beneath each footplate. When depressed by $2.5-\mathrm{mm}$ movement, the hinge closed a microswitch. A Grason-Stadler 901B noise generator supplied a white masking noise to each box through a $6.4-\mathrm{cm}$ speaker located on the ceiling of the apparatus. White noise $(80 \mathrm{~dB})$ was continuously present during intertrial intervals. The CS consisted of a 10 -sec offset of the masking noise.

A 28-V dc ceiling light was used as the feedback stimulus. Each shuttlebox was placed inside ventilated styrofoam ice chests and was located in a darkened room with additional white noise $(80 \mathrm{~dB})$ provided through a $20-\mathrm{cm}$ speaker mounted on the ceiling of the test room. Programming was controlled by two-channel punched tapes and $28-\mathrm{V}$ dc electromechanical relay circuitry. Scrambled shock was provided by two GrasonStadler Model 700 shockers. Recording of all events was accom- plished by two Scientific Prototype Model CR2D cumulative recorders and electromechanical counters. All programming and recording equipment was located in an adjoining room.

\section{Procedure}

Subjects were randomly assigned to one of the two shuttleboxes and trained to an avoidance criterion of $50 \%$ or better of the second 100 trials of daily 200 trial sessions. A trial consisted of a 10-sec CS (white noise offset in the unit's speaker). If the subject failed to cross the center section of the apparatus, a .5-sec 1.0-mA inescapable shock followed. CS and US offset were coterminus. The maximum trial length was $10.5 \mathrm{sec}$, and the interval from one CS onset to the next was $30 \mathrm{sec}$.

An avoidance response consisted of the initial depression of the footplate, resulting in the illumination of a 1-sec feedback light and preclusion of shock on that trial. Only one feedback signal per trial was possible regardless of the number of times the animal crossed the midsection of the apparatus. An avoidance response precluded shock but did not affect CS termination.

Following acquisition, subjects were matched with partners who were within $10 \%$ of their final acquisition levels. Matched pairs of subjects were randomly divided into two feedback stimulus contingencies: response-contingent feedback or yoked feedback. Half of each group was then divided into two extinction groups: one that never received shock and one that received shock following each CS. Thus, the four extinction groups were as follows: (1) response-contingent or operant feedback and no shock (OpNS), (2) yoked feedback and no shock (YNS), (3) operant feedback and shock on every trial (OpS), and (4) yoked feedback and shock on every trial (YS). Subjects were always tested in the same shuttleboxes that they were trained in, and shuttleboxes were taken into consideration for the assignment to the four extinction conditions. Thus, two extinction groups, OpS and YS, received $100 \%$ CS-US pairings, while the other two groups, OpNS and YNS, never received shock in extinction.

Day 1 of extinction consisted of 100 trials of acquisition for a warm-up, followed by 100 trials of extinction. The subsequent 3 days consisted of 200 extinction trials/day. Data were analyzed in 100-trial blocks.

\section{RESULTS}

\section{Acquisition}

In order to confirm that there were no differences due to assignment of animals to the four extinction groups, an analysis of variance was performed on the final avoidance responses during the last 100 trials of acquisition. No significant differences were found $[F(3,12)=.76, p>.05]$. A second analysis of variance for uncorrelated samples was performed on the avoidance responses from the 100 warm-up trials prior to the start of extinction. Again, no significant differences were found among the subjects' performances $[F(3,12)=.04$, $\mathrm{p}>.05]$. These data confirmed that subjects assigned to each of the four contingencies had attained comparable terminal acquisition performances.

\section{Extinction}

A 2 by 2 by 7 analysis of variance (responsecontingent vs. yoked feedback by shock vs. no shock by trial blocks) was performed on the extinction responses with repeated measures on the feedback condition (due to the yoked design) and on the trial blocks. The 


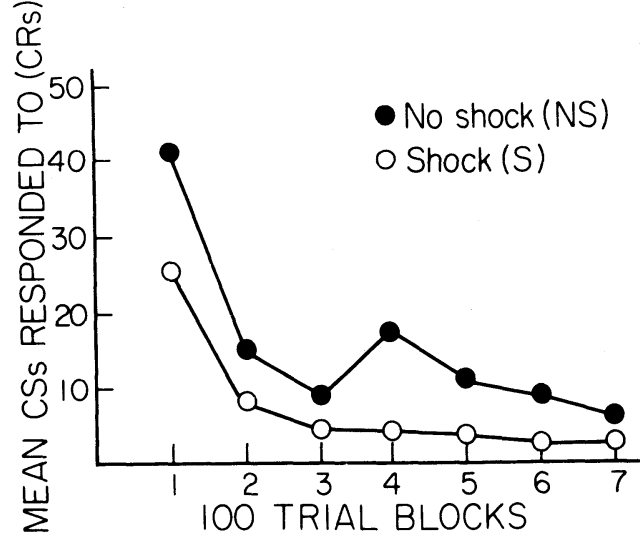

Figure 1. Mean conditioned responses for shock and nonshock groups combined during extinction of avoidance behavior.

results revealed a significant effect of the shock condition $[F(1,10)=7.50, p<.01]$. As can be seen in Figure 1, the no-shock groups showed higher maintained responding than the shock groups. This effect remained throughout the course of extinction, but was most marked on Blocks 1 and 4 . A significant effect of trial blocks was also found $[F(6,60)=53.37, p<.01]$, demonstrating that responding in fact significantly declined over trials. In addition, a significant Extinction Condition by Blocks interaction was obtained $[F(6,60)$ $=5.54, \mathrm{p}<.01]$, which, as shown in Figure 1, demonstrated that the no-shock groups showed greater resistance to extinction than the shock groups over the 700 trials. Although no significant main effect of feedback was found $(\mathrm{F}<1.0)$, a significant Feedback Condition by Blocks interaction was obtained $[\mathrm{F}(6,60)$ $=38.83, \mathrm{p}<.01]$, demonstrating the superiority of response-contingent feedback over yoked feedback sometime during the 700 trials of extinction.

From Figure 2, it can be readily observed that operant subjects were superior to their yoked partners only during the first 100 trials of extinction.

Finally, there was a highly significant three-way interaction $[F(6,60)=55.48, p<.01]$, suggesting that at least one of the four groups differed significantly from the others. As can be seen in Figure 3, operant subjects that received no shock demonstrated greater maintained responding, especially during the first 100 trials of extinction.

Subsequent $t$ tests on the differences between simple main effects (Cochran \& Cox, 1957) were performed on the data from each of the trial blocks. The results showed that the only significant differences occurred on the first trial block between the OpNS group and the remaining three [OpNS vs. YNS: $\mathrm{t}(10)=2.37, \mathrm{p}<.05$; OpNS vs. OpS: $\mathrm{t}(10)=2.77, \mathrm{p}<.02 ;$ OpNS vs. YS: $t(10)=2.37, p<.05]$. Thus, the OpNS group responded at a higher level than any of the other three groups on the first trial block, following which all groups extinguished responding to about the same level.

\section{DISCUSSION}

The superiority of the group receiving responsecontingent feedback in combination with no shock (OpNS) suggests an interpretation of extinction of avoidance behavior in terms of generalization decrement. During terminal acquisition, avoidance behavior typically exposes the subjects to very few shocks. Thus, when a no-shock extinction procedure is instituted, OpNS subjects are performing under conditions that most resemble successful avoidance, that is, response-contingent feedback and no shock. In contrast, the conditions present in extinction for YNS subjects differ from the OpNS animals only in the manner in which the feedback signal is presented. Wtih the response/feedback signal contingency disrupted, responding rapidly diminishes due to generalization decrement. Traditional as well as more contemporary versions of two-factor theory would have some difficulty with these results. According to the traditional view, the major source of reinforcement for maintained responding is still present, that of US nonoccurrence. A modified view, which regards both CS termination and/or feedback signal as safety signals for shock-free periods that permit feedback to acquire conditioned fear-inhibitory properties, would probably not expect differential effects of responseproduced vs. yoked feedback signal, since both are equally reliable predictors of the nonoccurrence of shock. Similarly, a cognitive view would require the assumption that stimulus-

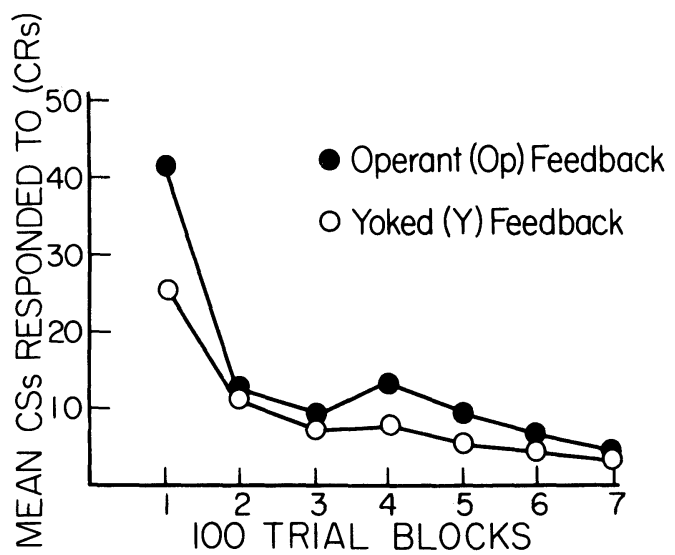

Figure 2. Mean conditioned responses for operant and yoked groups combined during extinction of avoidance behavior.

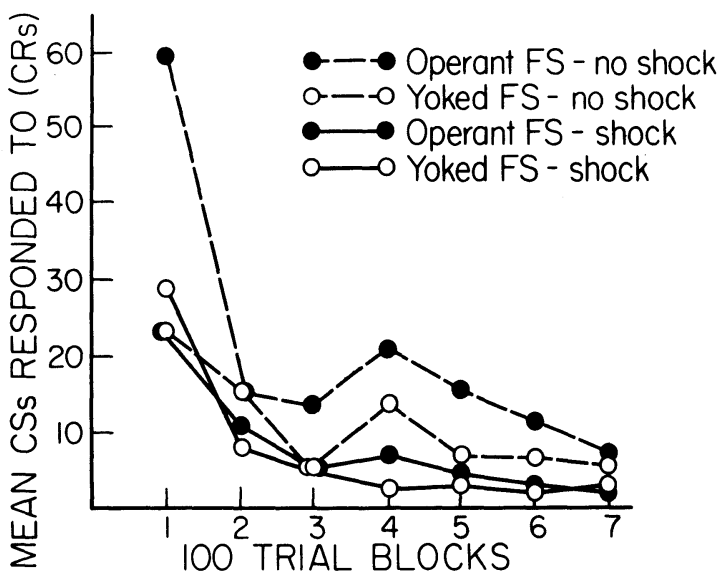

Figure 3. Resistance to extinction of avoidance for each of the four treatment groups. 
outcome and response-outcome expectancies differ in associative value.

For those subjects receiving shock on all trials, the feedback signal may have become a cue signaling the imminence rather than the omission of shock. The more likely the subject is to respond (as is typically the case early in extinction), the more likely it is to receive a feedback signal just prior to shock onset. Thus, for the OpS subjects, response-produced feedback may acquire conditioned aversive properties due to adventitious punishment. For the YS subjects, all responses are ineffective, that is, they can neither produce feedback nor preclude shock. Thus, not only is there a generalization decrement due to disruption of the response/feedback signal contingency, but there is further suppression of responding due to an increase in the frequency of shocks.

The present results may at first appear to conflict with those of Jackson and Scheuer (1972) and Scheuer and Sutton (1973), who found greater resistance to extinction under shock than under no-shock conditions. However, for their subjects, the presence of shock was the only relevant cue common to acquisition and extinction; neither warning signal termination nor feedback was permitted for either group during either phase of their experiments. Furthermore, their use of a leverpress as the avoidance response may have minimized the importance of proprioceptive feedback cues that typically result from a response such as running.

We are of the opinion that the results of the present experiment confirm the notion of the functional similarity between response-produced feedback and response-produced CS termination and disagree with Cicala and Owen (1976), who have claimed that warning signal termination and feedback serve different functions during extinction of avoidance behavior. The additivity that Cicala and Owen obtained during extinction, as well as the differential extinction rates for warning signal termination and feedback signal groups, may have been due not to the functional dissimilarities between each type of contingency, but rather, to differences in salience of each of the cues used separately and in combination. We do, however, agree with the conclusion reached by Galvani and Twitty (1978), who argue that response-produced feedback is an effective reinforcer in the absence of warning signal termination. Whether both of these events derive their reinforcing characteristics through a mechanism of fear reduction or they provide information about the nonoccurrence of shock remains a question. Although no direct comparison between warning signal termination and feedback was made in the present study, when we parallel the results obtained by Hartley (1968) with our own, the functional similarity between response-produced warning signal termination and feedback is apparent. While Hartley has noted that the avoidance contingency (shock vs. no shock) interacts with response-contingent $\mathrm{CS}$ termination to prolong extinction, we have obtained similar effects by employing response-produced feedback. The only major distinction between Hartley's design and our own relates to our inclusion of a yoked procedure to insure that the critical variable prolonging extinction was not merely the presence of feedback, but its dependence on responding.

\section{REFERENCES}

Bolles, R. C., \& Grossen, N. E. Effects of an informational stimulus on the acquisition of avoidance behavior in rats. Journal of Comparative and Physiological Psychology, 1969, 68, 90-99.

Bolles, R. C., Moot, S. A., \& Grossen, N. E. The extinction of shuttlebox avoidance. Learning and Motivation, 1971, 2, 324-333.

Cicala, G. A., \& Owen, J. W. Warning signal termination and a feedback signal may not serve the same function. Learning and Motivation, 1976, 7, 356-367.

Cochran, W. G., \& Cox, G. M. Experimental designs. New York: Wiley, 1957.

D'Amato, M. R., Fazzaro, J., \& Etkin, M. Anticipatory responding and avoidance discrmination as factors in avoidance conditioning. Journal of Experimental Psychology, 1968, 77, 41-47.

Galvani, P. F., \& TwitTy, M. T. Effects of intertrial interval and exteroceptive feedback duration on discriminative avoidance acquisition in the gerbil. Animal Learning \& Behavior, 1978, 6, 166-173.

HARTLEY, D. L. Sources of reinforcement in learned avoidance. Journal of Comparative and Physiological Psychology, 1968, 66, 12-16.

Jackson, M. C.. JR., \& Scheuer, C. A comparison of two procedures for breaking the response-reinforcement contingency in discriminated barpress avoidance. Psychonomic Science, 1972, 29, 14-16.

Katzev, R. D., \& Henderson, R. W. Effects of exteroceptive feedback stimuli on extinguishing avoidance responses in Fischer rats. Journal of Comparative and Physiological Psychology, 1971, 74, 66-74.

Mowren, O. H., \& Jones, L. V. Habit strength as a function of the pattern of reinforcement. Journal of Experimental Psychology, 1945, 35, 292, 311.

Mowrer, O. H., \& Lamoreaux, R. R. Fear as an intervening variable in avoidance conditioning. Journal of Comparative and Physiological Psychology, 1946, 39, 29-50.

Scheuer, C., \& Sutton, C. O. Discriminative versus motivational interpretations of avoidance extinction: Extensions to learned helplessness. Animal Learning \& Behavior, 1973, 1, 193-197.

Sheffield, V. F. Extinction as a function of partial reinforcement and distribution of practice. Journal of Experimental Psychology, 1949, 39, 511-526.

Solomon, R. L., \& BRUSH, E. S. Experimentally derived conceptions of anxiety and aversion. In M. R. Jones (Ed.), Nebraska Symposium on Motivation, 1956, 4, 212-305.

(Received for publication March 26, 1979.) 